\title{
Efficient Dynamic Analysis of Foundation via a Coupled Axisymmetric SBFEM-3D FEM
}

\author{
Mojtaba ASLMAND ${ }^{1}$ \\ Iradj Mahmoudzadeh KANI ${ }^{2}$ \\ Mehmet Cemal GENES ${ }^{3}$
}

\begin{abstract}
An axisymmetric scaled boundary finite element method for the elastodynamic analysis of 3D layered systems is derived in frequency domain. The general three-dimensional problem of unbounded domain can be divided into a number of independent two-dimensional problems and a pseudo three-dimensional analysis can be performed. The aforementioned analysis offers considerable savings in terms of storage and computation cost when compared with a full three-dimensional analysis. A new coupled strategy is proposed in order to match the axi-symmetrically modeled unbounded far field to a general 3D finite element model of the near field. The derived formulation is implemented for frequency domain analysis of foundation embedded in or resting on the surface of 3D layered homogenous or inhomogeneous soil deposits over rigid bedrock. The dynamic response calculated using the proposed method is compared with analytical or numerical solutions. Numerical examples demonstrate the accuracy and high efficiency of the new proposed method.
\end{abstract}

Keywords: Axisymmetric scaled boundary finite element method, layered soil, dynamic soil-structure interaction, Fourier series, dynamic stiffness.

\section{INTRODUCTION}

Dynamic response of heavy and embedded structures such as nuclear reactors, turbine towers and offshore wind plants, liquid-storage tanks and high-rise buildings are affected by the interaction between the structures and their foundations. These problems are known as soil-

Note:

- This paper has been received on July 25, 2018 and accepted for publication by the Editorial Board on November 12, 2018.

- Discussions on this paper will be accepted by September 30, 2019.

- https://dx.doi.org/10.18400/tekderg.447721

1 University of Tehran, School of Civil Engineering, Tehran, Iran - maslmand@ut.ac.ir https://orcid.org/0000-0003-1241-094X

2 University of Tehran, School of Civil Engineering, Tehran, Iran - imkani@ut.ac.ir https://orcid.org/0000-0003-0124-7868

3 Eastern Mediterranean University, Civil Engineering Department, Famagusta, North Cyprus cemal.genes@emu.edu.tr - https://orcid.org/0000-0002-9052-7361 
structure interaction (SSI) problems. The main goal of many related engineering studies is to develop SSI models, which are reliable and easy to implement.

One of the major challenges in this area is the lack of a precise and consistent template to model radiation damping of the unbounded soil domain. Consequently, the conventional finite element method is not directly applicable, since outgoing waves are reflected at the artificial boundaries of the finite element mesh. Over the past five decades, various approaches have been introduced in order to overcome this problem [1-6].

Most of the common approaches to model dynamic soil-structure interaction problems in layered media can be classified as the boundary element method [7-9], the thin layer method (TLM) [10-15] or approximate methods based on physical models [16-22] and semianalytical methods [23, 24].

The boundary element method is a popular tool for the solution of dynamic problems in unbounded domains since it is based on the use of fundamental solutions, which satisfies the radiation condition explicitly. Green's functions for layered media are complex and computationally expensive [25-27].

A widely used method for dynamic analysis of layered media is the thin-layer method which has mainly been applied to plane and cylindrical problems [28]. It is a semi-analytical approach based on the coupling of an axisymmetric finite element formulation with an analytical solution in the wave number and frequency-domain in the direction of wave propagation. The latter is obtained by discretization in the direction of layering.

Interesting from a practical point of view, simplified or physical models lead to approximate solutions. Cone models [29] have been introduced for the analysis of rigid foundations by one-dimensional wave propagation formulation in the frequency-domain. Semi-analytical solutions are based on a discretization of the footing-subsoil interface and the element-wise use of Green's functions [24,30].

A relatively recent semi-analytical approach, which shows certain parallels to the thin-layer method, is the scaled boundary finite element method (SBFEM) [31]. It is particularly suitable for the analysis of dynamic problems in unbounded domains. The SBFEM combines some important advantages of the finite element method (FEM) and the boundary element method (BEM). When this method is applied, the spatial dimension of the problem is reduced by one eliminating the need for fundamental solution. The material anisotropy only affects the constitutive matrix and is implemented straightforwardly. A SBFE model of an unbounded domain can be coupled seamlessly with a finite element model of the near-field. The combined formulation has been used for dynamic 2D and 3D SSI problems in both the frequency domain and time domain [32-37].

The original SBFEM [31] is based on the use of a scaling centre, somehow the reference point for assessing the visibility of the complete boundary. It defines a transformation of the geometry such that the problem can be solved numerically in the circumferential directions and analytically in the radial direction. It can be used for the description of $2 \mathrm{D}$ or $3 \mathrm{D}$ halfspace or full-space unbounded domains.

A modified version of the SBFEM for two-dimensional domains with parallel boundaries [38], for three-dimensional prismatic domains [39] and for axisymmetric domain [40] exists. All three versions of the SBFEM, however, are not used to model a truly three-dimensional 
layered system. Recently, a Modified SBFEM for the analysis of dynamic problems in threedimensional layered continua is derived [28]. The latter is based on the use of a scaling line, rather than a scaling centre and it fully couples the 3D FEM with 2D SBFEM. In order to reduce the computational cost and accuracy of the solution, the formulation has been modified to an axisymmetric SBFEM, in which only a line discretization is needed and the solution could be obtained for each term of the Fourier series separately [41].

The recently derived formulation by Aslmand et al. [41] for coupling a semi-analytical axisymmetric far field formulation and a 3D finite element model is available. The coupling is done by the aid of Fourier coefficients and the time domain developments were achieved by FFT. The proposed novel SBFEM for 3D layered media is considerably different from the original SBFEM. In combination with a finite element model of the near field, it can be used efficiently for the dynamic analysis of flexible three-dimensional foundations of arbitrary shape embedded in or resting on the surface of layered soil deposits over rigid bedrock.

The radiation condition states that there are not any incoming waves. For special cases the radiation requirement can be expressed as a local formulation, such as Sommerfeld radiation condition [42] for the Hemholtz's equation in infinite homogeneous domains. Generalized radiation conditions involve elastodynamic Green's functions for the semi-infinite solid and for the layered half-space which have been established in studies of Guzina et al. [43] and Madyarov and Guzina [44]. Nevertheless, in this study, the radiation condition at infinity is satisfied automatically by using the SBFEM $[31,45]$ to model the far field with axisymmetric formulation.

This paper focuses on frequency domain examples by coupling between axisymmetric SBFEM and 3D FEM. The further outline of this paper is as follows. In the first section, the assumptions in this paper to analyze three-dimensional foundation-soil systems are summarized. Then, the formulation of axisymmetric SBFEM for unbounded layered media is summarized. The coupling to a 3D finite element model of the near field is addressed in the next section, and finally frequency domain analysis of various layered soil-foundation systems are held for verification and present the efficiency of the newly developed coupling method.

\section{PROBLEM DEFINITION}

The substructure method has been used for the analysis of structures with flexible foundations embedded in a layered soil (Figure 1). The material properties of layer $j$ which is unbounded in horizontal direction has been defined as shear modulus $\left(G_{j}\right)$, Poisson's ratio $\left(v_{j}\right)$, mass density $\left(\rho_{j}\right)$ and thickness $\left(d_{j}\right)$ and a constant damping ratio $D$ for all layers.

In order to have an axisymmetric geometry for the interface of near field/far field, the near field shall be defined by a cylinder containing the arbitrary shaped foundation and a certain part of the irregular soil. Within this context, the near field/far field interface is denoted as $S$ as depicted in Figure 1. For modeling of the near field, traditional 3D FEM technique is adopted. On the other hand, the far field is analyzed through utilization of an axisymmetric SBFEM where this approach has been previously implemented and validated in the study conducted by Aslmand et al. [41]. In the next section, executed axisymmetric SBFEM analysis is summarized. 
It is assumed that dynamic loads are concentrated either in the near field or on the structure system only and hence outwardly propagating waves including surface and interfacial waves are generated.

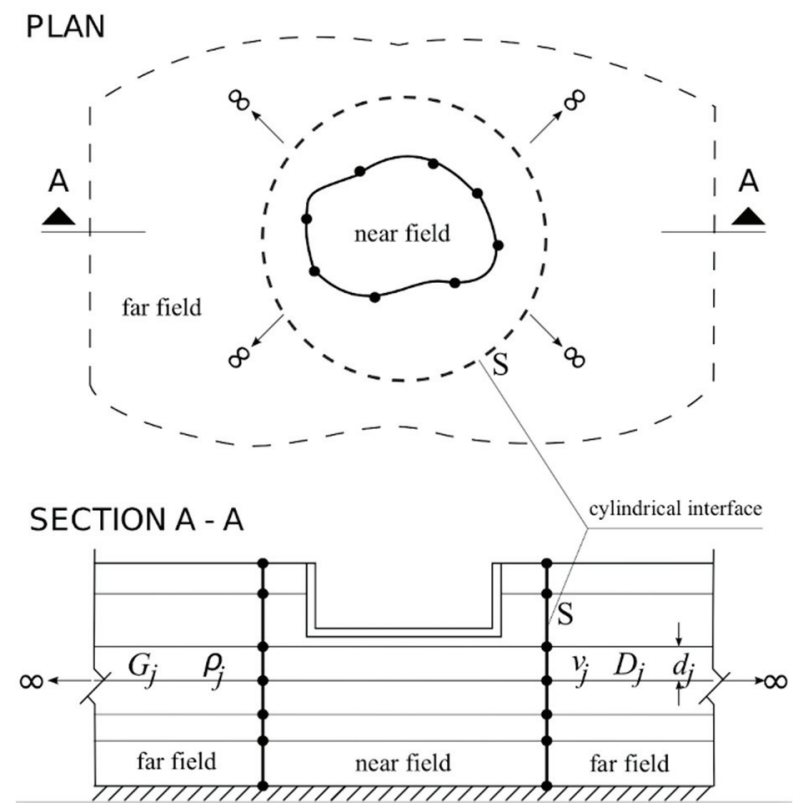

Figure 1 - 3D soil-structure system with foundation embedded in layered soil deposit resting on rigid bedrock

\subsection{Axisymmetric Scaled Boundary Finite Element Method}

\subsubsection{Scaled Boundary Transformation of the Geometry}

The modified SBFEM uses a coordinate system, which scales a defining curve S relative to a scaling line. As shown in Figure 2, in axisymmetric SBFEM the scaling line is identical to the axis of symmetry (the $\hat{z}$-axis). The radial direction $\xi$ is described by a series of parallel rays, which are perpendicular to the scaling line. Radial coordinate $\xi$ is equal to 0 and 1 at the intersection with the $\hat{z}$-axis and the line $\mathrm{S}$, respectively. But also, $\xi$ could be considered greater than $1(0 \leq \xi<\infty)$.

Due to consideration of axisymmetric geometry, using the advantage of cylindrical coordinates $(r, z, \theta)$ in the far field is more convenient (Figure 2).

The governing equation for the linear elastodynamics in cylindrical coordinate state,

$\overline{\mathrm{L}}^{T} \sigma+\omega^{2} \rho \mathrm{u}=0$ 
where $\boldsymbol{\sigma}, \mathbf{u}$ and $\overline{\mathbf{L}}$ are stresses, displacements and the equilibrium operator in cylindrical coordinate, respectively. Assuming linear elasticity, the stress-strain relationship can be expressed according to Hooks' law

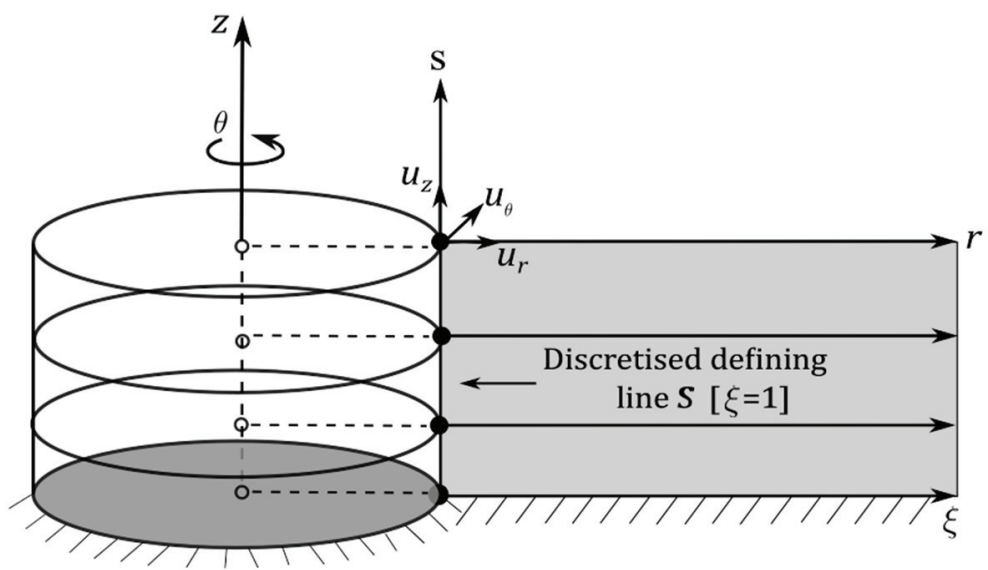

Figure 2 - Definition of SBFE local coordinates for an axisymmetric unbounded domain

$\sigma=\mathrm{D} \varepsilon$

where, $\mathbf{D}$ is constitutive matrix and

$\varepsilon=\mathrm{Lu}$

where $\mathbf{L}$ is a differential operator in cylindrical coordinate [41]. The approximate solution of Eq. (1) is obtained using a Fourier series in the circumferential $\theta$ direction and using conventional one-dimensional finite-element shape functions along the boundary $\mathrm{S}$ which leads to a series of ordinary differential equations in terms of the radial coordinate $\xi$ [41]. Hence, a solution is written in the form

$\left\{\begin{array}{l}u_{r}(\xi, s, \theta) \\ u_{z}(\xi, s, \theta) \\ u_{\theta}(\xi, s, \theta)\end{array}\right\}=\sum_{n=0}^{\infty}\left\{\mathbf{F}_{u}^{s}(\theta, n) \mathbf{N}(s) \mathbf{u}^{s}(\xi, n)+\mathbf{F}_{u}^{a}(\theta, n) \mathbf{N}(s) \mathbf{u}^{a}(\xi, n)\right\}$

where,

$\mathbf{F}_{u}^{s}(\theta, n)=\left[\begin{array}{ccc}\cos n \theta & 0 & 0 \\ 0 & \cos n \theta & 0 \\ 0 & 0 & -\sin n \theta\end{array}\right], \quad \mathbf{F}_{u}^{a}(\theta, n)=\left[\begin{array}{ccc}\sin n \theta & 0 & 0 \\ 0 & \sin n \theta & 0 \\ 0 & 0 & \cos n \theta\end{array}\right]$ 
$\mathbf{N}(s)$ is the shape functions matrix corresponding to the discretization of $s$, and $\mathbf{u}^{s}(\xi, n)$ and $\mathbf{u}^{a}(\xi, n)$ represent the variation of the nodal displacement in the $\xi$ direction for the symmetric and anti-symmetric Fourier terms, respectively.

Mapping to the scaled boundary co-ordinate system, the linear operator $\mathbf{L}$ can be expressed as

$\mathbf{L}=\mathbf{b}^{1}(s) \frac{\partial}{\partial r}+\mathbf{b}^{2}(s) \frac{\partial}{\partial z}+\mathbf{b}^{3}(s) \frac{1}{r}+\mathbf{b}^{4}(s) \frac{1}{r} \frac{\partial}{\partial \theta}+\mathbf{b}^{5}(s) \frac{1}{r} \frac{\partial}{\partial \theta}$

Where $\mathbf{b}^{1}(s)$ to $\mathbf{b}^{5}(s)$ are defined in [41].

Substituting Eqs. (4), (6) and (3) in Hooks' law (2), the stress can be expressed more clearly [41].

The virtual work principle for the dynamic case (in absence of body loads) reads

$$
\begin{gathered}
\int_{V} \delta \boldsymbol{\varepsilon}(\xi, s, \theta)^{\mathrm{T}} \boldsymbol{\sigma}(\xi, s, \theta) \mathrm{dV}+\int_{V} \delta \mathbf{u}(\xi, s, \theta) \rho \ddot{\mathbf{u}}(\xi, s, \theta) \mathrm{dV}- \\
\int_{S} \int_{0}^{2 \pi} \delta \mathbf{u}(s, \theta)^{\mathrm{T}} \mathbf{t}(s, \theta)|J(s)| \mathrm{d} \theta \mathrm{d} s=0
\end{gathered}
$$

The first term denotes the virtual work of internal strain energy. The second term is the virtual work of inertia forces and last but not least, the third term is the virtual work of external forces such as surface tractions $\mathbf{t}(s, \theta)$ acting on the discretized boundary (It is assumed that all of the surface tractions are in the near field). As aforementioned, the contribution of the three terms to the virtual work is already derived in the study of Aslmand et al. [41] to obtain the SBFE equations in displacement for an unbounded layered axisymmetric medium as given in Eqs. (8)-(11). It is a must for these equations to satisfy each symmetric and antisymmetric component of the Fourier series.

$$
\begin{aligned}
& \mathbf{P}^{s}(n)=\mathbf{E}^{0 s}(n) \mathbf{u}^{s}(n)_{, \xi}+\left(\mathbf{E}^{1 s}(n)^{\mathrm{T}}+\mathbf{E}^{3 s}(n)\right) \mathbf{u}^{s}(n) \\
& \mathbf{E}^{0 s}(n) \xi \mathbf{u}^{s}(\xi, n)_{, \xi \xi}+\left(\mathbf{E}^{0 s}(n)-\mathbf{E}^{1 s}(n)+\mathbf{E}^{1 s}(n)^{\mathrm{T}}+\xi \mathbf{E}^{3 s}(n)-\xi \mathbf{E}^{3 s}(n)^{\mathrm{T}}\right) \mathbf{u}^{s}(\xi, n)_{, \xi} \\
& +\left(\frac{1}{\xi} \mathbf{E}^{2 s}(n)+\mathbf{E}^{3 s}(n)-\mathbf{E}^{4 s}(n)-\mathbf{E}^{4 s}(n)^{\mathrm{T}}-\xi \mathbf{E}^{5 s}(n)\right) \mathbf{u}^{s}(\xi, n)+ \\
& \omega^{2} \mathbf{M}_{0}^{s}(n) \xi \mathbf{u}^{s}(\xi, n)=0 \\
& \mathbf{P}^{a}(n)=\mathbf{E}^{0 a}(n) \mathbf{u}^{a}(n)_{, \xi}+\left(\mathbf{E}^{1 a}(n)^{\mathrm{T}}+\mathbf{E}^{3 a}(n)\right) \mathbf{u}^{a}(n) \\
& \mathbf{E}^{0 a}(n) \xi \mathbf{u}^{a}(\xi, n)_{, \xi \xi}+\left(\mathbf{E}^{0 a}(n)-\mathbf{E}^{1 a}(n)+\mathbf{E}^{1 a}(n)^{\mathrm{T}}+\xi \mathbf{E}^{3 a}(n)-\xi \mathbf{E}^{3 a}(n)^{\mathrm{T}}\right) \mathbf{u}^{a}(\xi, n)_{, \xi} \\
& +\left(\frac{1}{\xi} \mathbf{E}^{2 a}(n)+\mathbf{E}^{3 a}(n)-\mathbf{E}^{4 a}(n)-\mathbf{E}^{4 a}(n)^{\mathrm{T}}-\xi \mathbf{E}^{5 a}(n)\right) \mathbf{u}^{a}(\xi, n)+ \\
& \omega^{2} \mathbf{M}_{0}^{a}(n) \xi \mathbf{u}^{a}(\xi, n)=0
\end{aligned}
$$

The solution of Eqs. (9) and (11) must be sought for each term of the Fourier Series independently. Eqs. (9) and (11) are the scaled boundary finite element equation in 
displacement for each term of Fourier series in an unbounded layered system. A particular solution for $u(\xi, n)\}$ is less interesting compared to a general solution for the dynamic stiffness matrix which relates the amplitude of coupling forces to the amplitudes of coupling.

The significance of this is that the system of equations decouples into pairs of equations for each symmetric and anti-symmetric term in the Fourier series representing the applied loading and displacement. Should a particular Fourier term in the loading series be zero, the corresponding term in the displacement series will also be zero. Thus, if the loading series only contains a single Fourier term, only a single set of equations need to be solved. Should the loading series contain more than one term, the set of equations for each term can be solved independently, and the resulting displacements and stresses superposed [40].

With the intention of obtaining equations in dynamic stiffness, the linear second-order differential Eqs. (9) and (11) in displacement are transformed into equivalent nonlinear firstorder differential equations as in Ref. [28], Eq. (60). After the derivation, Equations for the dynamic stiffness matrix of unbounded medium is obtained,

$$
\begin{aligned}
& \left(\mathbf{S}^{s}(\omega, \xi, n)+\mathbf{E}^{1 s}(n)+\xi \mathbf{E}^{3 s}(n)^{\mathrm{T}}\right) \mathbf{E}^{0 s}(n)^{-1}\left(\mathbf{S}^{s}(\omega, \xi, n)+\mathbf{E}^{1 s}(n)^{\mathrm{T}}+\xi \mathbf{E}^{3 s}(n)\right) \\
& -\xi \mathbf{S}^{s}(\omega, \xi, n)_{, \xi}-\xi\left(\mathbf{E}^{4 s}(n)+\mathbf{E}^{4 s}(n)^{\mathrm{T}}\right)-\xi^{2} \mathbf{E}^{5 s}(n)+\omega^{2} \xi^{2} \mathbf{M}^{0 s}(n)=0 \\
& \\
& \left(\mathbf{S}^{a}(\omega, \xi, n)+\mathbf{E}^{1 a}(n)+\xi \mathbf{E}^{3 a}(n)^{\mathrm{T}}\right) \mathbf{E}^{0 a}(n)^{-1}\left(\mathbf{S}^{a}(\omega, \xi, n)+\mathbf{E}^{1 a}(n)^{\mathrm{T}}+\xi \mathbf{E}^{3 a}(n)\right) \\
& -\xi \mathbf{S}^{a}(\omega, \xi, n)_{, \xi}-\xi\left(\mathbf{E}^{4 a}(n)+\mathbf{E}^{4 a}(n)^{\mathrm{T}}\right)-\xi^{2} \mathbf{E}^{5 a}(n)+\omega^{2} \xi^{2} \mathbf{M}^{0 a}(n)=0
\end{aligned}
$$

Eqs. (12) and (13) are the scaled boundary finite element equations in dynamic stiffness for an unbounded axisymmetric layered medium.

The nonlinear first-order differential equation with respect to $\xi$ is solved numerically using a Runge-Kutta scheme for a given frequency $\omega^{*}$ instead. An initial value is required to start the numerical integration scheme. It is calculated in the following using an asymptotic expansion of the dynamic stiffness $\mathbf{S}^{\infty}\left(\omega^{*}, \xi\right)$ with respect to the radial coordinate $\xi$.

\subsubsection{Asymptotic Expansion of Dynamic Stiffness in $\xi$}

The unknown dynamic stiffness matrix $\mathbf{S}^{\infty}(\xi, n)$ for both symmetric and anti-symmetric is expressed as a power series in $(\xi)$ with decreasing exponent. In the following the superscript $s$ and $a$ and the series number $n$ has been omitted for convenience

$$
\mathbf{S}^{\infty}(\xi) \approx(\xi)^{1} \mathbf{C}_{\infty}+(\xi)^{0} \mathbf{K}_{\infty}+\sum_{j=1}^{m} \frac{1}{(\xi)^{j}} \mathbf{A}_{\mathbf{j}}
$$

The eigenvalue problem

$$
\mathbf{M}^{0} \boldsymbol{\Phi}=\mathbf{E}^{0} \boldsymbol{\Phi} \mathbf{m}^{0}, \quad \boldsymbol{\Phi}^{\mathrm{T}} \mathbf{E}^{0} \boldsymbol{\Phi}=\mathbf{I}, \quad \boldsymbol{\Phi}^{\mathrm{T}} \mathbf{M}^{0} \boldsymbol{\Phi}=\mathbf{m}^{0}
$$

is used to transform Eqs. (12) and (14) into 


$$
\begin{aligned}
& \left(\mathbf{s}^{\infty}(\xi)+\mathbf{e}^{1}+\xi \mathbf{e}^{3^{\mathrm{T}}}\right)\left(\mathbf{s}^{\infty}(\xi)+\mathbf{e}^{1^{\mathrm{T}}}+\xi \mathbf{e}^{3}\right)-\xi \mathbf{s}^{\infty}(\xi)_{, \xi}-\mathbf{e}^{2}-\xi\left(\mathbf{e}^{4}+\mathbf{e}^{4^{\mathrm{T}}}\right) \\
& -\xi^{2} \mathbf{e}^{5}+\omega^{2} \xi^{2} \mathbf{m}^{0}=0
\end{aligned}
$$

With

$$
\mathbf{s}^{\infty}(\xi)=\boldsymbol{\Phi}^{\mathrm{T}} \mathbf{S}^{\infty}(\xi) \boldsymbol{\Phi}, \quad \mathbf{e}^{j}=\boldsymbol{\Phi}^{\mathrm{T}} \mathbf{E}^{j} \boldsymbol{\Phi}, \quad j=1,2, \ldots, 5
$$

Note that both $\mathbf{M}^{0}$ and $\mathbf{E}^{0}$ are positive definite values, thus all eigenvalues are real positive. Thus, Eq. (14) is transformed analogously,

$\mathbf{s}^{\infty}(\xi) \approx(\xi)^{1} \mathbf{c}_{\infty}+(\xi)^{0} \mathbf{k}_{\infty}+\sum_{j=1}^{m} \frac{1}{(\xi)^{j}} \mathbf{a}_{j}$

Where

$$
\mathbf{c}_{\infty}=\boldsymbol{\Phi}^{\mathrm{T}} \mathbf{C}_{\infty} \boldsymbol{\Phi}, \quad \mathbf{k}_{\infty}=\boldsymbol{\Phi}^{\mathrm{T}} \mathbf{k}_{\infty} \boldsymbol{\Phi}, \quad \boldsymbol{a}_{j}=\boldsymbol{\Phi}^{\mathrm{T}} \mathbf{A}_{j} \boldsymbol{\Phi}
$$

The derivative with respect to $\mathbf{s}^{\infty}(\xi), \xi$ is expressed as

$$
\mathbf{s}^{\infty}(\xi)_{, \xi} \approx \mathbf{c}_{\infty}-\sum_{j=1}^{m} \frac{j}{(\xi)^{j}} \mathbf{a}_{j}
$$

The power series Eq. (18) and its derivative Eq. (20) are substituted in Eq. (16). The coefficients $\mathbf{c}_{\infty}, \mathbf{k}_{\infty}$ and $\mathbf{a}_{j}$ are calculated equating terms in the resulting formulation corresponding to decreasing powers of $\xi$ to zero. The quadratic term yields

$\mathbf{c}_{\infty} \mathbf{c}_{\infty}+\mathbf{e}^{3^{\mathrm{T}}} \mathbf{c}_{\infty} \mathbf{e}^{3}+\mathbf{e}^{3^{\mathrm{T}}} \mathbf{e}^{3}-\mathbf{e}^{5}+\omega^{2} \mathbf{m}^{0}=0$

Eq. (21) is an algebraic Riccati equation for the coefficient $\mathbf{C}_{\infty}$ [46].

Its solution involves the Schur decomposition of an associated Hamiltonian matrix $\mathbf{H}$. The eigenvalues occur in pairs $\pm \lambda$ where $\lambda$ contains all eigenvalues with negative real part or positive imaginary part, if $\boldsymbol{\lambda}$ is purely imaginary. Complex eigenvalues with negative real parts correspond to modes which are decaying with $\xi \rightarrow \infty$ purely imaginary eigenvalues with positive imaginary part correspond to physically acceptable outwardly propagating modes. Only the Schur vectors associated with $\pm \boldsymbol{\lambda}$ are used to construct $\mathbf{C}_{\infty}$. In doing so, the radiation condition is taken into account.

The linear term yields

$$
\begin{aligned}
& \left(\mathbf{c}_{\infty}+\mathbf{e}^{3^{\mathrm{T}}}\right) \mathbf{k}_{\infty}+\mathbf{k}_{\infty} \mathbf{c}_{\infty}+\mathbf{e}^{3}=-\left(\mathbf{c}_{\infty}+\mathbf{e}^{3^{\mathrm{T}}}\right) \mathbf{e}^{1^{\mathrm{T}}}-\mathbf{e}^{1}\left(\mathbf{c}_{\infty}+\mathbf{e}^{3}\right) \\
& +\mathbf{c}_{\infty}+\left(\mathbf{e}^{4}+\mathbf{e}^{4^{\mathrm{T}}}\right)
\end{aligned}
$$


Eq. (22) is a Lyapunov equation for the coefficient $\mathbf{k}_{\infty}$ with a symmetric coefficient matrix $\mathbf{c}_{\infty}$. Its solution is computed using Schur decomposition and subsequently solving a Sylvester equation [47].

The constant term yields

$$
\left(\mathbf{c}_{\infty}+\mathbf{e}^{3^{\mathrm{T}}}\right) \mathbf{a}_{1}+\mathbf{a}_{1}\left(\mathbf{c}_{\infty}+\mathbf{e}^{3}\right)=-\left(\mathbf{k}_{\infty}+e^{1}\right)\left(\mathbf{k}_{\infty}+\mathbf{e}^{1^{\mathrm{T}}}\right)+\mathbf{e}^{2}
$$

Eq. (23) is again a Lyapunov equation for the coefficient $\mathbf{a}_{1}$, which is solved analogously to Eq. (22).

Equations for higher order terms $\mathbf{a}_{\mathrm{j}}, \mathrm{j}>1$, can be derived in an analogous manner. The initial value of the dynamic stiffness is constructed evaluating Eq. (24) for a high but finite value $\xi_{h}$.

$\mathbf{S}^{\infty}\left(\omega^{*}, \xi_{h}\right) \approx\left(\boldsymbol{\Phi}^{-1}\right)^{\mathrm{T}} \xi_{h} \mathbf{C}_{\infty}+\mathbf{k}_{\infty}+\sum_{j=1}^{m} \frac{j}{\left(\xi_{h}\right)^{j}} \mathbf{a}_{\mathbf{j}} \boldsymbol{\Phi}^{-1}$

In order to consider material damping in the formulation given above, Linear hysteretic material damping is incorporated to the proposed method by using a complex-valued shear modulus $G^{*}$ instead of the real-valued quantity $G$,

$G^{*}=(1+i 2 D) G$

In Eq. (25) the symbols $i$ and $D$ denote the imaginary unit and the damping ratio, respectively.

\section{COUPLING OF 3D FEM-AXISYMMETRIC SBFEM}

The interior domain is modeled by using an ordinary 3D finite element approach. The coupling between the finite element model of the near field and the axisymmetric scaled boundary finite element model of the far field is not straightforward, provided that the 2D near field/ far field interface surface shall be presented by the 1D discretization of a line regarding axisymmetric SBFEM.

To couple axisymmetric and non-axisymmetric domains each mesh node in the circumference of the cylinder enclosing the near field (interface) should be considered to find the corresponding columns of the unbounded stiffness matrix. The $r^{\text {th }}$ column of the unbounded stiffness matrix represents the nodal load vector to enforce a certain deformation configuration, where the $r^{\text {th }}$ nodal dof is unity and the rest are zero. The stiffness matrix of the interface will be constructed from this concept. For instance, supposition a unit value of displacement in $r$ direction of point $i$ as is depicted in Figure (3(a)), the variation of displacement around the circumferential line contain node $i$ would be a linear function which has the value of unity at node $i$ and zero elsewhere (Figure 3(b)). Note that the variation of displacement in $z$ direction would be satisfied with the shape functions of linear elements on the line of discretization matching with the finite element ones (here the mesh is restricted to 8-node brick elements for the near field and linear elements for the far field). The Fourier series of the corresponding function named $\mathrm{u}(\theta)$ is written as: 


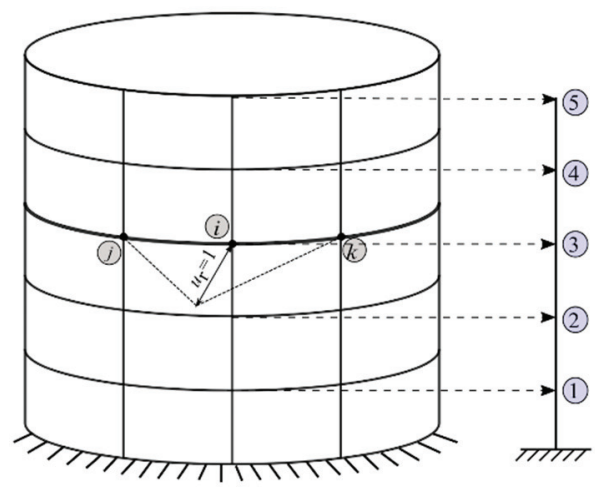

(a)

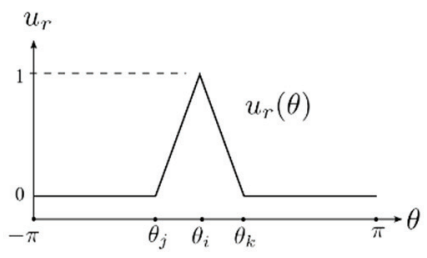

(b)

Figure 3 - (a) Interface of near field/far field (b) Linear variation of displacement around circumference due to the imposed unit displacement

$u(\theta)=\frac{a_{0}}{2}+\sum_{k=1}^{n}\left(a_{k} \cos k \theta+b_{k} \operatorname{sink} \theta\right)$

where the Fourier coefficients $a_{k}, k=0,1,2, \ldots, n$ and $b_{k}, k=1,2, \ldots, n$ are given by

$a_{k}=\frac{1}{\pi} \int_{-\pi}^{\pi} u(\theta) \cos k \theta d \theta, \quad b_{k}=\frac{1}{\pi} \int_{-\pi}^{\pi} u(\theta) \sin k \theta d \theta$

Each of these Fourier coefficients corresponds to the symmetric and anti-symmetric displacement of node $i$ for a particular Fourier term on the discretized line. Having the corresponding stiffness matrix for each particular Fourier series, one could find the loading contribution as:

$f^{s}(\omega, k)=\mathbf{S}^{s}(\omega, k) \mathbf{u}_{k}^{s}(\omega, k), \quad \mathbf{u}_{k}^{s}=\left\{0,0 \ldots a_{k} \ldots 0\right\}^{\mathrm{T}} \quad k=0,1,2, \ldots$

$f^{a}(\omega, k)=\mathbf{S}^{a}(\omega, k) \mathbf{u}_{k}^{a}(\omega, k), \quad \mathbf{u}_{k}^{a}=\left\{0,0 \ldots b_{k} \ldots 0\right\}^{\mathrm{T}}$

where $\mathbf{u}_{n}^{s}$ are $\mathbf{u}_{n}^{a}$ the displacement vectors of our discretized line which have only one non zero element on the corresponding degrees of freedom of node $i$ (See Fig. 3). Superposing the results of forces for each term of Fourier series, the total forces for each particular position $(\theta, s)$ becomes obtainable with the help of the following equation:

$\left\{\begin{array}{l}F_{r}(\theta, s) \\ F_{z}(\theta, s) \\ F_{\theta}(\theta, s)\end{array}\right\}=\sum_{n=0}^{\infty}\left\{\mathbf{F}_{u}^{s}(\theta, n) \mathbf{N}(s) \mathbf{f}^{s}(n)+\mathbf{F}_{u}^{a}(\theta, n) \mathbf{N}(s) \mathbf{f}^{a}(n)\right\}$

Where $\mathbf{F}_{u}^{s}(\theta, n)$ and $\mathbf{F}_{u}^{a}(\theta, n)$ were given in Eq. (5), and $\mathbf{N}(s)$ is the one-dimensional finiteelement shape functions. 
The Eq. (29) should be set for each node on the near field/far field interface to obtain one column of total stiffness matrix due to the particular deformation in node $i$. This procedure shall be repeated for all degrees of freedom for all the nodes to obtain the total stiffness matrix.

In the frequency-domain, the interior FEM model is represented by the following finite element equation:

$$
\left(\mathbf{K}-\omega^{2} \mathbf{M}\right) \widehat{\mathbf{u}}=\widehat{\mathbf{P}}
$$

with the static stiffness matrix $\mathbf{K}$, the mass matrix $\mathbf{M}$, the vector of unknown displacement amplitudes $\widehat{\mathbf{u}}$ and the vector of nodal forces $\widehat{\mathbf{P}}$. The system matrices and the nodal vectors are partitioned such that all degrees of freedom corresponding to the soil-structure interface are assembled in $\widehat{\mathbf{u}}_{b}$, whereas all remaining interior degrees of freedom are denoted as $\widehat{\mathbf{u}}_{1}$.

$$
\left(\left[\begin{array}{cc}
\mathbf{K}_{i i} & \mathbf{K}_{i b} \\
\mathbf{K}_{b i} & \mathbf{K}_{b b}
\end{array}\right]-\omega^{2}\left[\begin{array}{ll}
\mathbf{M}_{i i} & \mathbf{M}_{i b} \\
\mathbf{M}_{b i} & \mathbf{M}_{b b}
\end{array}\right]\right)\left[\begin{array}{c}
\widehat{\mathbf{u}}_{i} \\
\widehat{\mathbf{u}}_{b}
\end{array}\right]=\left[\begin{array}{c}
\widehat{\mathbf{P}}_{i} \\
\widehat{\mathbf{P}}_{b}
\end{array}\right]-\left[\begin{array}{c}
\mathbf{0} \\
\widehat{\mathbf{R}}_{b}
\end{array}\right]
$$

Here, the vector of nodal forces $\widehat{\mathbf{P}}$ is split into contributions due to external loads $\widehat{\mathbf{P}}_{\text {ex }}=$ $\left[\begin{array}{ll}\widehat{\mathbf{P}}_{i} & \widehat{\mathbf{P}}_{b}\end{array}\right]^{\mathrm{T}}$ and due to the coupling forces $\widehat{\mathbf{R}}_{b}$. The external loads are partitioned into the interior loads $\widehat{\mathbf{P}}_{i}$ and interface loads $\widehat{\mathbf{P}}_{\mathbf{b}}$. This is illustrated in Figure 4 . The dynamic stiffness matrix $\mathbf{S}^{\infty}\left(\omega^{*}, \xi=1\right)$ relates the amplitudes of the coupling forces to the amplitudes of the displacements at the near field/far field interface all in cylindrical coordinate. To obtain the coupling force vector $\widehat{\mathbf{R}}_{b}(\theta, s)$ in cylindrical coordinate

$$
\widehat{\mathbf{R}}_{b}(\theta, s)=\mathbf{T S}^{\infty}\left(\omega^{*}, \xi=1\right) \mathbf{T}^{\mathrm{T}} \widehat{\mathbf{u}}_{b}
$$

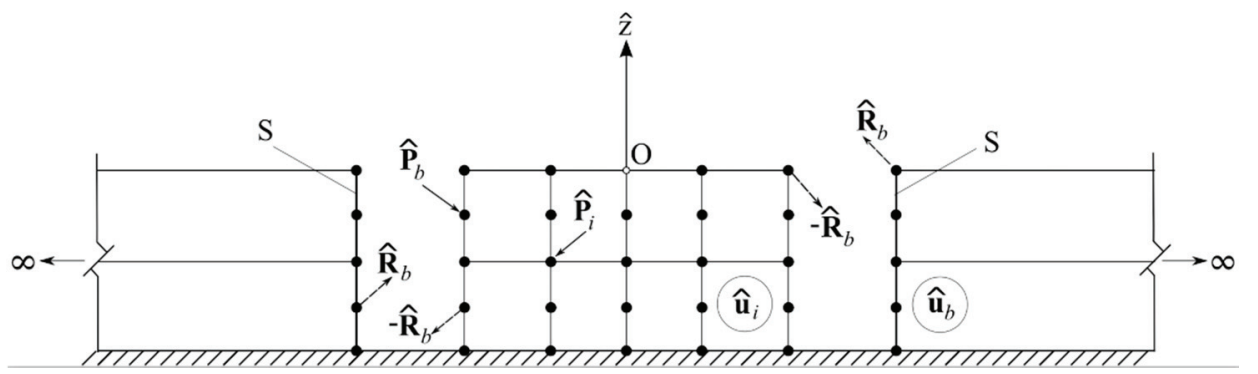

Figure 4 - FEM-SBFEM coupling: partitioning of degrees of freedom

Where the transformation matrix $\mathbf{T}$ is for converting cylindrical to Cartesian coordinate. Substituting Eq. (32) into Eq. (31)

$$
\left[\begin{array}{cc}
\mathrm{K}_{i i}-\omega^{2} \mathrm{M}_{i i} & \mathrm{~K}_{i b}-\omega^{2}\left[\mathrm{M}_{i b}\right] \\
\mathrm{K}_{b i}-\omega^{2} \mathrm{M}_{b i} & \mathrm{~K}_{b b}-\omega^{2} \mathrm{M}_{b b}+\mathrm{TS}^{\infty}\left(\omega^{*}, \xi=1\right) \mathrm{T}^{T}
\end{array}\right]\left[\begin{array}{l}
\hat{\mathrm{u}}_{i} \\
\hat{\mathrm{u}}_{b}
\end{array}\right]=\left[\begin{array}{l}
\widehat{\mathrm{P}}_{i} \\
\widehat{\mathrm{P}}_{b}
\end{array}\right]
$$


or

$\mathbf{S}_{G}(\omega) \widehat{\mathbf{u}}=\widehat{\mathbf{P}}_{e x}$

with the complex dynamic stiffness matrix $\mathbf{S}_{G}(\omega)$ of the coupled soil-structure system. Using Eq. (34), the displacement amplitudes of the near field due to time-harmonic external loads $\widehat{\mathbf{P}}_{e x}$ can be calculated.

\section{NUMERICAL EXAMPLES}

In this section, dynamic response of various foundations has been calculated with respect to the aforementioned derivation. A combined 3D FEM-Axisymmetric SBFEM program has been developed in the MATLAB programming language. In the solution procedure, 3D 8node isoparametric brick elements are used for the FE mesh and a conforming mesh of 2node linear elements are utilized for the SBFEM. The mesh needed for modeling the structure are first created in ANSYS commercial software [48] and then imported into the program as input. The numerical results obtained using the proposed method is later compared to reference solutions. Stiffness coefficients are presented in numerical results which is defined by:

$\mathbf{S}\left(a_{0}\right)=\mathbf{K}_{\text {stat }}\left(\mathbf{k}\left(a_{0}\right)+i a_{0} \mathbf{c}\left(a_{0}\right)\right)$

In Eq. (35), the symbols $\mathbf{K}_{\text {stat }}, \mathbf{k}\left(a_{0}\right)$ and $\mathbf{c}\left(a_{0}\right)$ denote the static stiffness coefficient and the frequency-dependent spring and damping coefficient, respectively. The dimensionless frequency $\mathrm{a}_{0}$ is defined as

$a_{0}=\omega \frac{r_{0}}{c_{s}}$

where $r_{0}$ is a typical physical dimension of the foundation and $c_{\mathrm{s}}$ is the shear wave velocity, $c_{s}=\sqrt{G / \rho}$.

\subsection{Rigid Circular Foundation on Homogeneous Soil Layer}

A rigid, massless circular foundation of radius $r_{0}$ resting on a homogeneous soil layer of thickness $d=3 r_{0}$ and Poisson's ratio $v=1 / 3$ is considered. The influence of the discretization on the static stiffness coefficient computed using the proposed method is studied. For the particular problem considered here, only the first two terms of the Fourier series is needed. Only the first term $(n=0)$ is necessary for the analysis of vertical vibration (symmetric case) or torsional vibration (antisymmetric case), and only the second term $(n=1)$ is needed for the study of horizontal or rocking vibration. The vertical, horizontal, rocking or torsional static stiffness coefficients of the rigid foundation computed using different meshes are summarized in Table 1. Four of these meshes are shown in Figure 5 for illustration purpose. 
Good agreement between these reference solutions [30] and the numerical results is obtained modeling the near field with only 6 finite elements (discretization 2). Using this coarse mesh, the static stiffness coefficients calculated using the proposed method differ from the reference solutions by $8 \%, 8 \%, 9 \%$ and $23 \%$ for vertical, horizontal, rocking and torsional excitation, respectively. Table 1 provides the computed static stiffness coefficients that converge to the values given by Gazetas when the mesh is refined. Using discretization 4 (1430 finite elements, 12 scaled boundary elements), the vertical, horizontal, rocking and torsional stiffness coefficients obtained numerically differ from the reference solutions by only $4 \%$, $1 \%, 1 \%$ and $11 \%$ respectively. This confirms that the derivation of the proposed axisymmetric SBFEM and the novel coupling method for three-dimensional layered systems is correctly implemented.
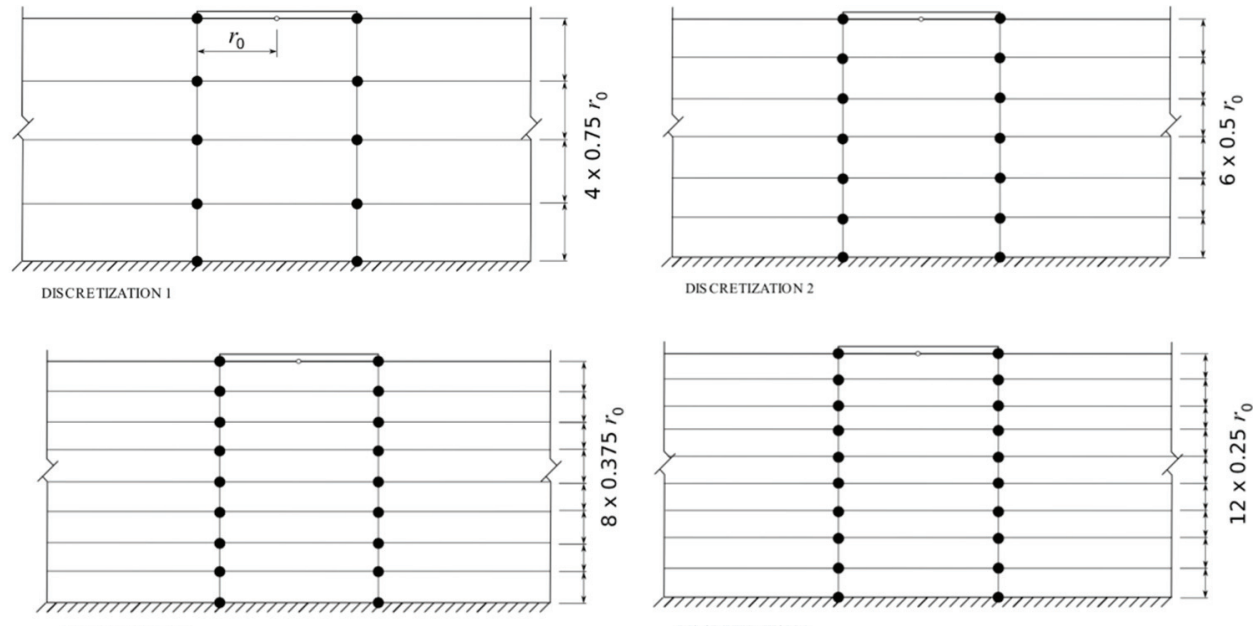

DISCRETZATION 3

DISCRETIZATION 4

Figure 5 - Rigid circular foundation resting on homogeneous soil over rigid bedrock:

Discretization 1 to 4

Table 1 - Convergence of static stiffness coefficients with decreasing mesh size

\begin{tabular}{|c|c|c|c|c|c|}
\hline \multicolumn{2}{|c|}{ Discretization } & \multirow{2}{*}{$\begin{array}{c}\text { Vertical } \\
n=0 \\
\mathbf{k}_{\text {stat.v }} \\
{\left[G_{0} r_{0}\right]}\end{array}$} & \multirow{2}{*}{$\begin{array}{c}\text { Horizontal } \\
n=1 \\
\mathbf{k}_{\text {stat }, \mathbf{h}} \\
{\left[G_{0} r_{0}\right]}\end{array}$} & \multirow{2}{*}{$\begin{array}{c}\text { Rocking } \\
n=1 \\
\mathbf{k}_{\text {stat, }} \\
{\left[G_{0} r_{0}{ }^{3}\right]}\end{array}$} & \multirow{2}{*}{$\begin{array}{c}\text { Torsion } \\
n=0 \\
\mathbf{k}_{\text {stat,t }} \\
{\left[\boldsymbol{G}_{0} \boldsymbol{r}^{\mathbf{3}}{ }_{0}\right]}\end{array}$} \\
\hline Nr. & $\begin{array}{l}\text { Number } \\
\text { of layers }\end{array}$ & & & & \\
\hline 1 & 4 & 9.541 & 6.430 & 4.907 & 7.221 \\
\hline 2 & 6 & 9.213 & 6.060 & 4.603 & 6.570 \\
\hline 3 & 8 & 9.052 & 5.870 & 4.449 & 6.258 \\
\hline 4 & 12 & 8.882 & 5.667 & 4.285 & 5.948 \\
\hline \multicolumn{2}{|c|}{$\begin{array}{l}\text { Reference solution } \\
\qquad[30]\end{array}$} & 8.560 & 5.600 & 4.222 & 5.333 \\
\hline
\end{tabular}




\subsection{Rigid Circular Foundation on Homogeneous Soil Layer under Dynamic Load}

Consider a rigid, massless circular foundation of radius $r_{0}$ which is resting on a homogeneous soil layer of thickness $d=r_{0}$ which the axisymmetric system is discretized using 102 -node scaled boundary elements and 4752 8-node finite elements, as shown in Figure 6.

The computed dynamic spring and damping coefficients are given by Figures 7 and 8 for horizontal and vertical, rocking and torsional motion, respectively.

The numerically obtained static stiffness coefficients $\mathrm{K}_{\text {stat }}$ are summarized in Table 2.

The dynamic stiffness coefficients computed using the proposed method are compared to reference solutions, where original results were previously published in Ref. [28, 29].

In general, the dynamic stiffness coefficients of layered soil resting over bedrock are strongly frequency-dependent. The first three eigen frequencies of the homogeneous layer corresponding to propagating P-waves or shear waves, respectively, are given in Table 3.

As depicted in Figures 7 and 8, the cutoff-frequency and higher Eigen frequencies are represented accurately using the proposed method. For all four modes of vibration, the damping coefficient is zero below the cutoff-frequency (that is 1.571). In general, the stiffness coefficients computed using the proposed method agrees well with the reference solutions. For the horizontal vibration which is obtained from the second term of series $(n=1)$, the curves produced using the proposed method are more different than those calculated using TLM. This could be due to the fact that solid elements produced by the ANSYS mesh generator [49] suffer from shear locking in numerical modelling while they have not been used in TLM. Moreover the components of coupled swaying-rocking stiffness could be defined differently with the results of TLM published in [29].
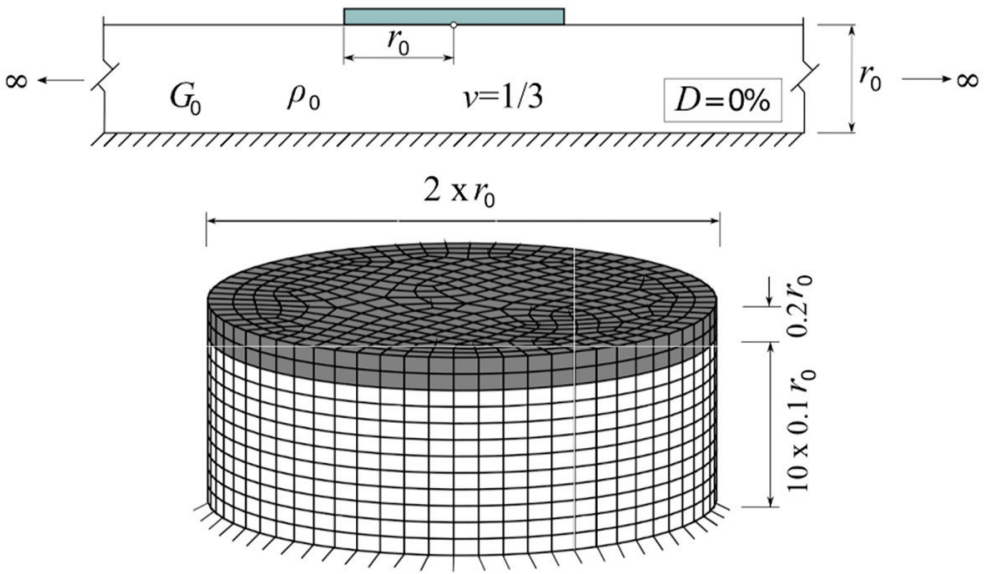

Figure 6 - Rigid circular foundation of radius $r_{0}$ resting on homogeneous soil layer of thickness $d=r_{0}$. System and discretization 

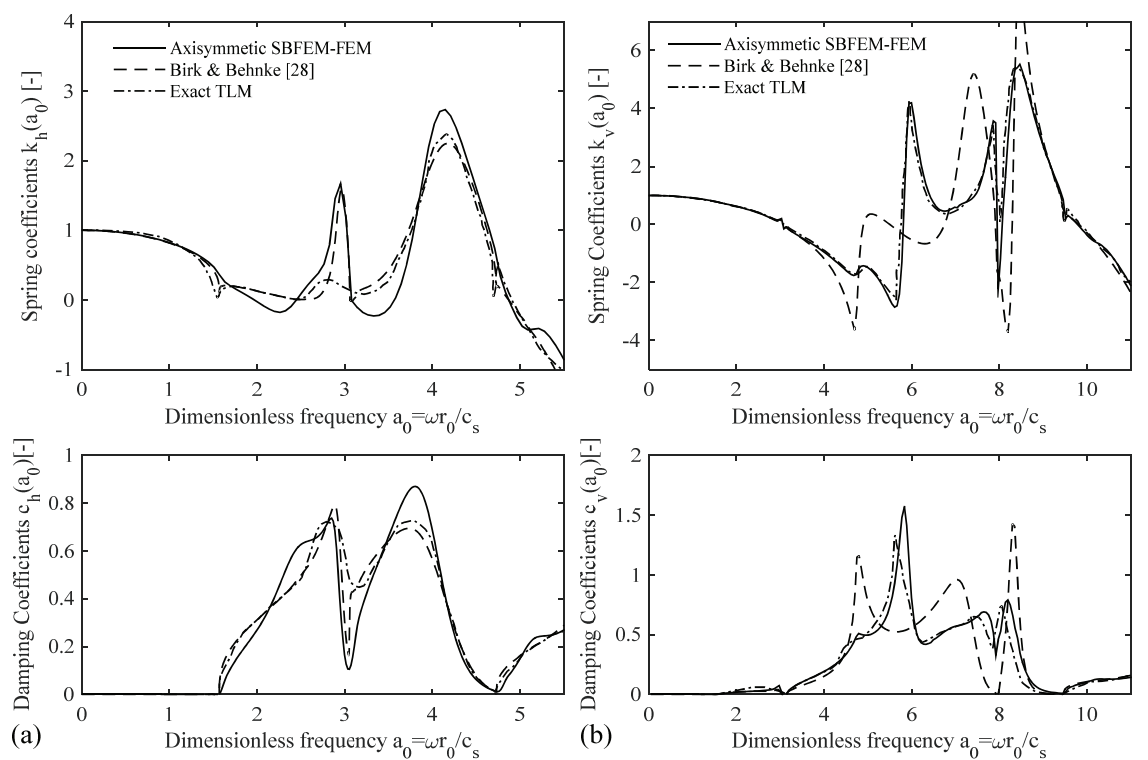

Figure 7 - Dynamic stiffness coefficients of rigid circular foundation of radius $r_{0}$ resting on a homogeneous soil layer of thickness $d_{0}$ : (a) horizontal $(n=1)$, (b) vertical $(n=0)$.
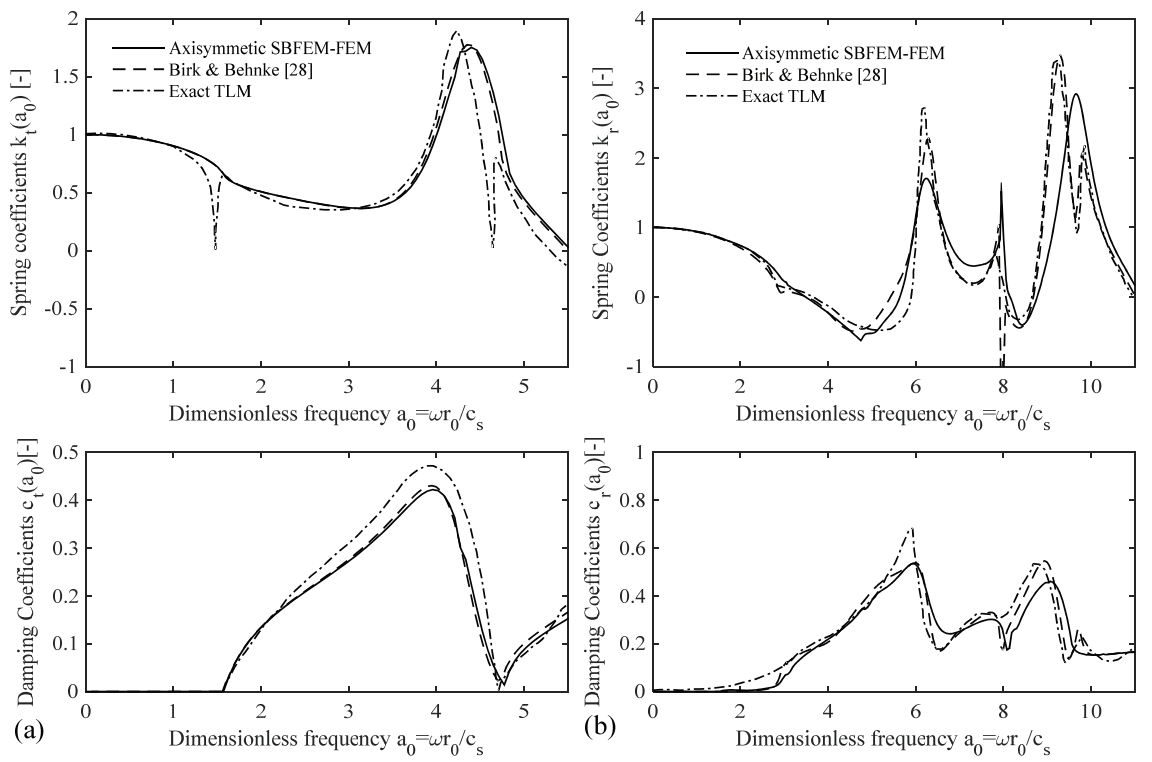

Figure 8 - Dynamic stiffness coefficients of rigid circular foundation of radius $r_{0}$ resting on a homogeneous soil layer of thickness $d_{0}$ : (a) torsion $(n=0)$, (b) rocking $(n=1)$. 
Table 2 - Static stiffness coefficients of rigid circular foundation of radius $r_{0}$ resting on homogeneous soil layer of thickness $d=r_{0}$

\begin{tabular}{|c|c|c|c|c|}
\hline & $\begin{array}{c}\text { Vertical } \\
\mathbf{k}_{\text {stat.v }}\end{array}$ & $\begin{array}{c}\text { Horizontal } \\
\mathbf{k}_{\text {stat }, \mathbf{h}}\end{array}$ & $\begin{array}{c}\text { Rocking } \\
\mathbf{k}_{\text {stat,r }}\end{array}$ & $\begin{array}{c}\text { Torsion } \\
\mathbf{k}_{\text {stat,t }}\end{array}$ \\
\hline Birk and Behnke [28] & $15.927 G_{0} r_{0}$ & $7.775 G_{0} r_{0}$ & $5.514 G_{0} r^{3}{ }_{0}$ & $6.027 G_{0} r^{3}{ }_{0}$ \\
\hline $\begin{array}{l}\text { Axisymmetric SBFEM- } \\
\text { FEM }\end{array}$ & $15.956 G_{0} r_{0}$ & $8.081 G_{0} r_{0}$ & $5.603 G_{0} r_{0}^{3}$ & $6.124 G_{0} r_{0}^{3}$ \\
\hline
\end{tabular}

Table 3 - Dimensionless eigen frequencies of homogeneous soil layer of depthd

\begin{tabular}{ccc}
\hline Eigen frequency $\boldsymbol{j}$ & $\begin{array}{c}\text { Shear waves } \boldsymbol{c}_{\boldsymbol{s}} \\
\text { horizontal and torsion }\end{array}$ & $\begin{array}{c}\text { P-waves } \boldsymbol{c}_{\boldsymbol{p}} \\
\text { vertical and rocking }\end{array}$ \\
\hline 1 (Cut-off frequency) & $\frac{1}{2} \pi=1.570$ & $\pi=3.141$ \\
2 & $\frac{3}{2} \pi=4.712$ & $3 \pi=9.424$ \\
3 & $\frac{5}{2} \pi=7.853$ & $5 \pi=15.707$ \\
\hline
\end{tabular}

\subsection{Rigid Circular Foundation on Inhomogeneous Soil Layer}

Considering a rigid circular foundation resting on a three parallel soil strata, as shown in Figure 9, the proposed method has been used to analyze a layered system with inhomogeneous material properties.

A constant hysteretic damping ratio of $D=0.05$ is assumed throughout the total thickness of the layer. This system has also been analyzed by Wolf and Preisig [18], and Birk and Behnke [28].

The soil depth has been discretized using 20 2-node scaled boundary elements and 73928 node finite elements. The corresponding mesh is shown in Figure 9.

Figure 10 shows the computed dynamic stiffness coefficients normalized using corresponding static stiffness.

The reference solutions shown in Figure 10 are the 'TLM exact' solutions published in Ref. [29] by thin-layer method and 3D-modifeid scaled boundary method published in Ref. [28].

The numerical results shown in Figure 10 agree well with the reference solutions.

Moreover, the explanation for deviation of horizontal vibration overlaps with the discussions made under section 4.2 . 


\subsection{Rigid Circular Foundation Embedded in Homogeneous Soil Layer}

A rigid circular foundation of radius $\mathrm{r}_{0}$ is embedded with depth $t=r_{0}$ in a homogeneous soil layer of thickness $d=3 r_{0}$ with Poisson's ratio $v=1 / 3$. Hysteretic damping of $D=0.05$ is assumed.
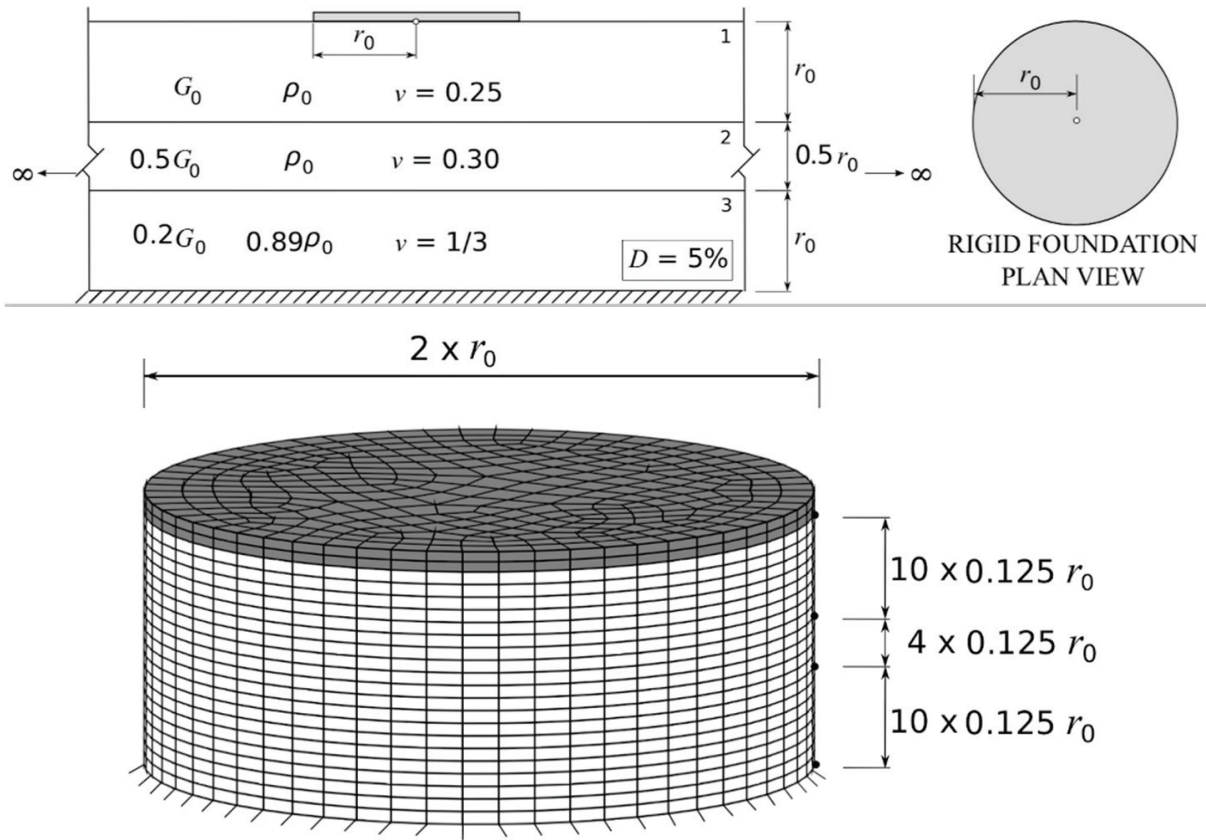

Figure 9 - Rigid circular foundation resting on inhomogeneous soil layer - discretization

The cylinder is modeled using 10 2-node scaled boundary elements and 440 8-nodes finite elements, as shown in Figure 11.

The static stiffness coefficients given in Refs. [18, 28] and the corresponding values calculated using the proposed method are summarized in Table 4.

The vertical and torsional dynamic stiffness coefficients which are calculated by using the proposed method are given in Figure 12.

These numerical results are compared to reference solutions which have been published in Refs. [18, 28]. The 'exact' solutions in Ref. [18] and in Figure 12 have been calculated by Emperador and Dominguez [50] using the boundary element method with a very fine discretization.

The agreement of the stiffness coefficient computed using the proposed method with the reference solutions based on the boundary element method and modified 3D SBFEM is found to be excellent. 
(a) horizontal
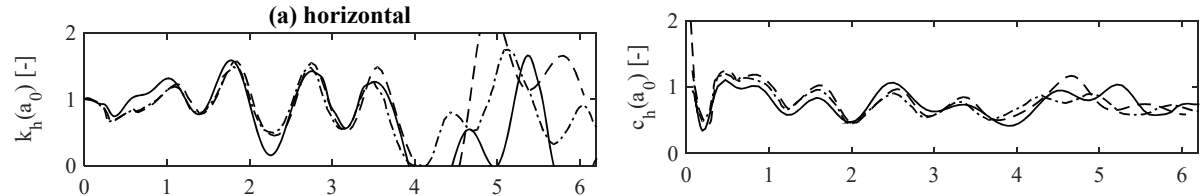

(b) vertical
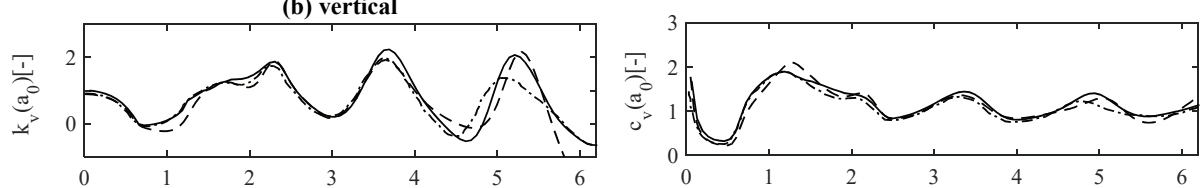

(c) rocking
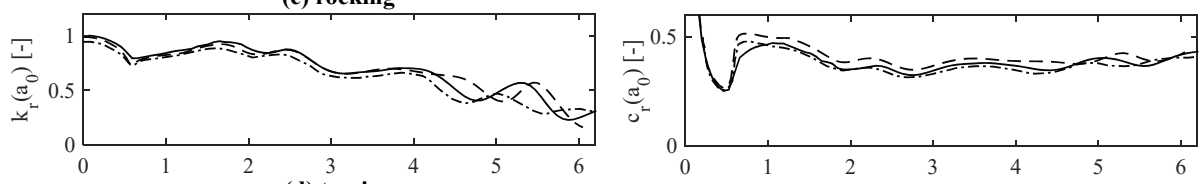

(d) torsion
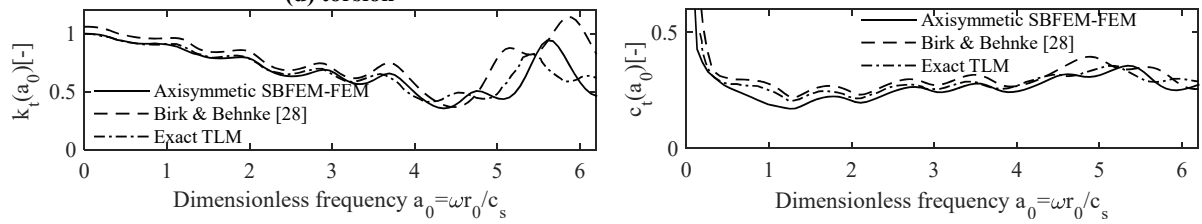

Figure 10 - Dynamic stiffness coefficients of rigid circular foundation of radius $r_{0}$ resting on inhomogeneous soil layer shown in Figure 9: (a) horizontal $(n=1)$, (b) vertical $(n=0)$,

(c) rocking $(n=1)$, (d) torsion $(n=0)$.

\subsection{Rigid Rectangular Foundation on Homogeneous Soil Layer}

To demonstrate the suitability of the proposed method for dynamic analysis of arbitrary shape foundation and study the effect of Fourier series terms, a rigid rectangular foundation of $L / B=2$ on a homogenous soil layer is considered, where $B$ and $L$ are half width and half length of foundation respectively. A constant Hysteretic damping of $D=0.05$ is assumed. The rectangular foundation enclosed by a cylinder of radius $=3 B$ which is the near field.
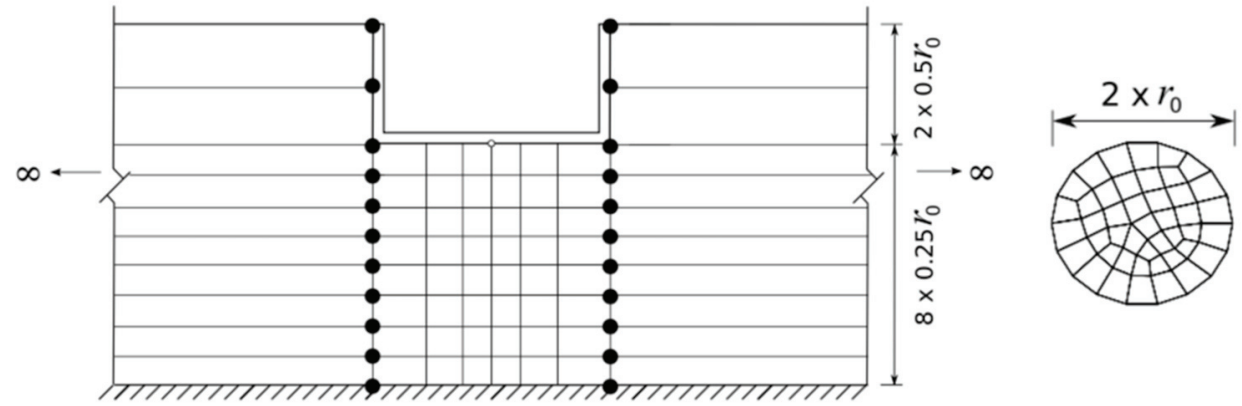

Figure 11 - Rigid circular foundation of radius $r_{0}$ embedded in homogeneous soil layer of thickness $d=3 r_{0}$ - dicretization 

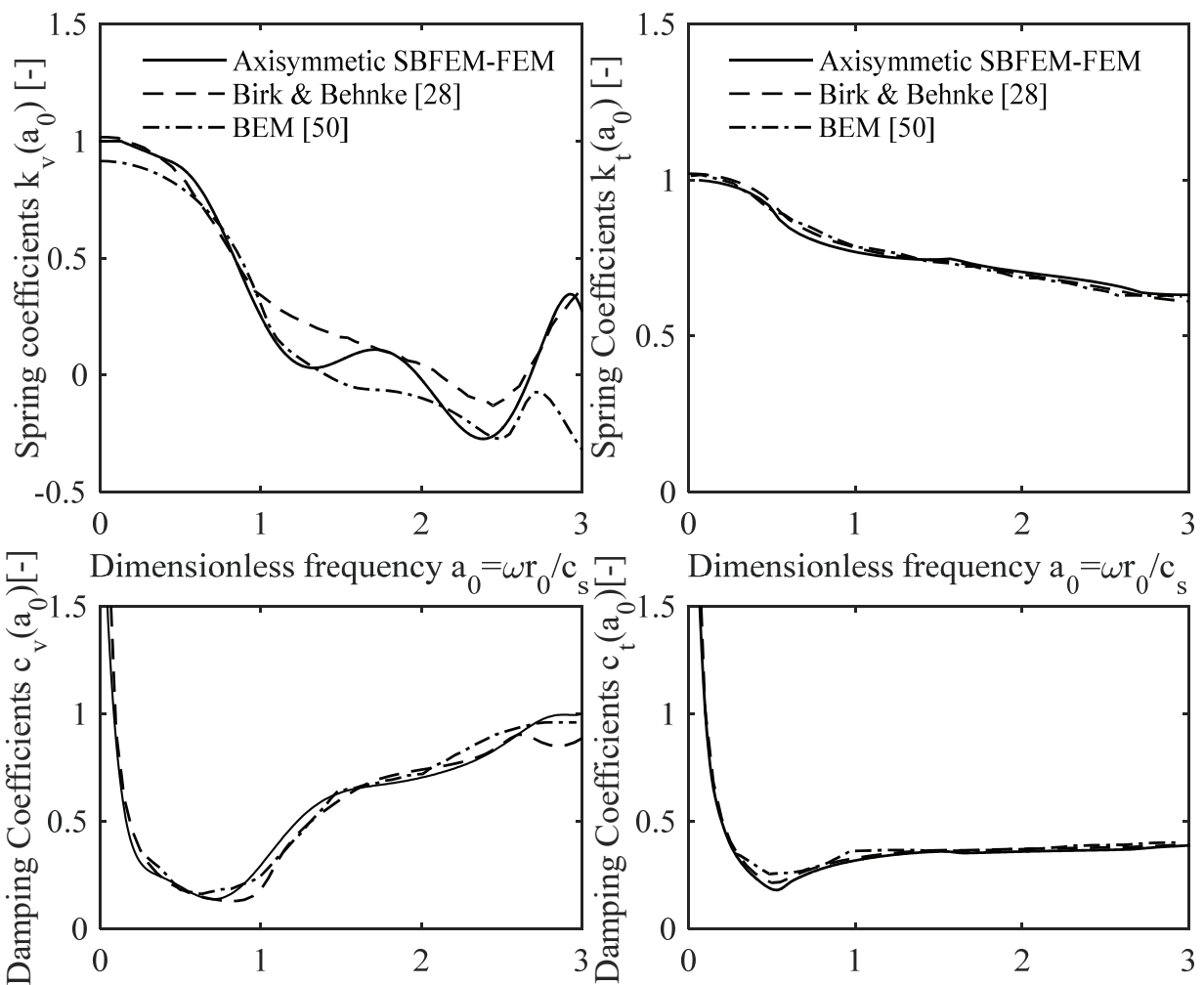

(a) Dimensionless frequency $\mathrm{a}_{0}=\omega \mathrm{r}_{0} / \mathrm{c}_{\mathrm{s}}$

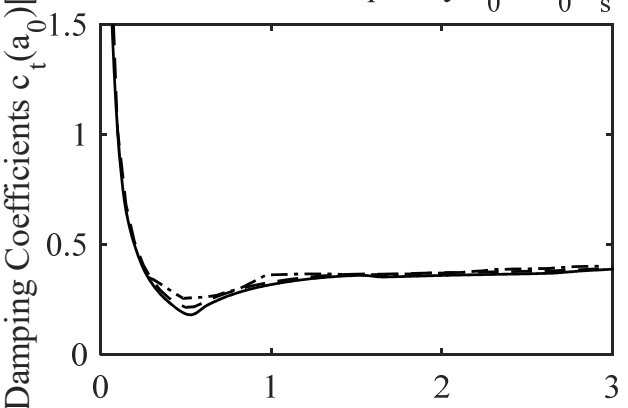

Figure 12 - Dynamic stiffness coefficients of rigid circular foundation of radius $r_{0}$ embedded with depth $t=r_{0}$ in homogeneous soil layer of depth $d=3 r_{0}$ : (a) vertical $(n=0)$, (b) torsion $(n=0)$

Two cases with different depth ratios $(h=4 B$ and $6 B)$ are considered. The boundaries of these two systems have been discretized using 16 and 24 scaled boundary elements respectively (Figure 13).

Table 4 - Static stiffness coefficients of rigid circular foundation of radius $r_{0}$ embedded with depth $r=r_{0}$ in homogeneous soil layer of thickness $d=3 r_{0}$

\begin{tabular}{ccc}
\hline & $\begin{array}{c}\text { Vertical } \\
\text { kstat, }\end{array}$ & $\begin{array}{c}\text { Torsion } \\
\text { kstat,s }\end{array}$ \\
\hline Axisymmetric SBFEM- FEM & $16.509 G_{0} r_{0}$ & $20.482 G_{0} r^{3}$ \\
Wolf and Preisig [17] & $16.14 G_{0} r_{0}$ & $20.22 G_{0} r^{3}{ }_{0}$ \\
Birk and Behnke [28] & $14.771 G_{0} r_{0}$ & $20.641 G_{0} r^{3}{ }_{0}$ \\
\hline
\end{tabular}


Three terms of series are needed to accurately represent the problem. Figure 14 shows the numerically obtained vertical impedances for $n=2$ along with values obtained by other researcher. The other results were obtained using the boundary solution method by Chow [51] and 3D frequency-dependent infinite element method by Seo et al., [52]. The proposed solution is found to be close to the reference values. Obtained results revealed that for a rigid rectangular foundation under vertical pressure, the Fourier series terms up to $n=2$ is sufficient.

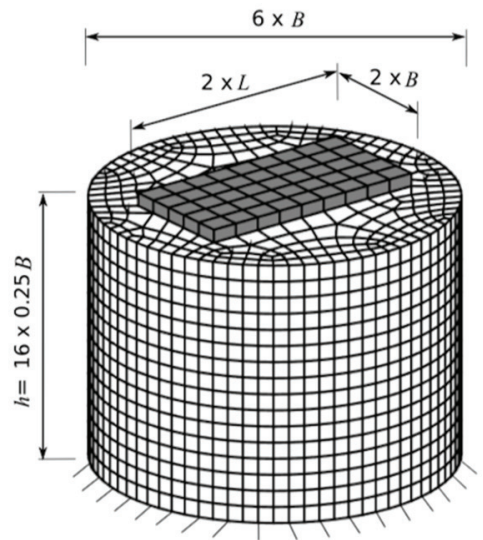

(a)

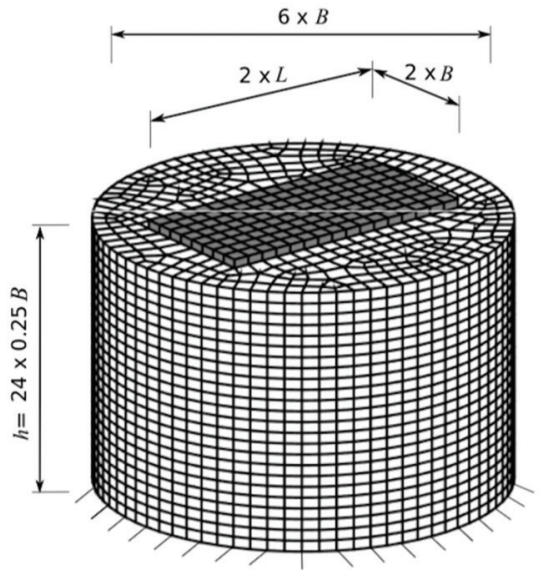

(b)

Figure 13 - Rigid rectangular foundation $(L=B=2)$ resting on homogeneous soil layer discretization: (a) $h / B=4$, (b) $h / B=6$

\section{CONCLUSION}

In this paper, an efficient computational model is presented for harmonic analysis of soilstructure interaction problem via a novel coupling of Axisymmetric SBFEM-3D FEM model.

It is based on deriving an axisymmetric scaled boundary finite element method for elastodynamic problems in three-dimensional layered media. Major differences both with respect to formulation and solution techniques occur with the original scaled boundary finite element method.

In the next step, the axisymmetric SBFEM formulation of unbounded domain will be coupled to the $3 \mathrm{D}$ finite element of the near field by a novel idea using the Fourier coefficients. The proposed method has been validated by calculating the static and dynamic stiffness of various foundation-soil systems.

The near field which can be of arbitrary complexity should be enclosed by a cylinder. Only the first term of series $(n=0)$ is needed for the vertical and torsional vibration of rigid circular foundation, while for the horizontal and rocking vibration, the second term $(n=1)$ should be used. The study demonstrate the need of three terms of Fourier series $(n=2)$ for a rigid rectangular foundation. 
To sum up, it can be said that the proposed method is very well suited for the frequency analysis of three-dimensional foundations of arbitrary shape, which are embedded in or resting on layered inhomogeneous soil.
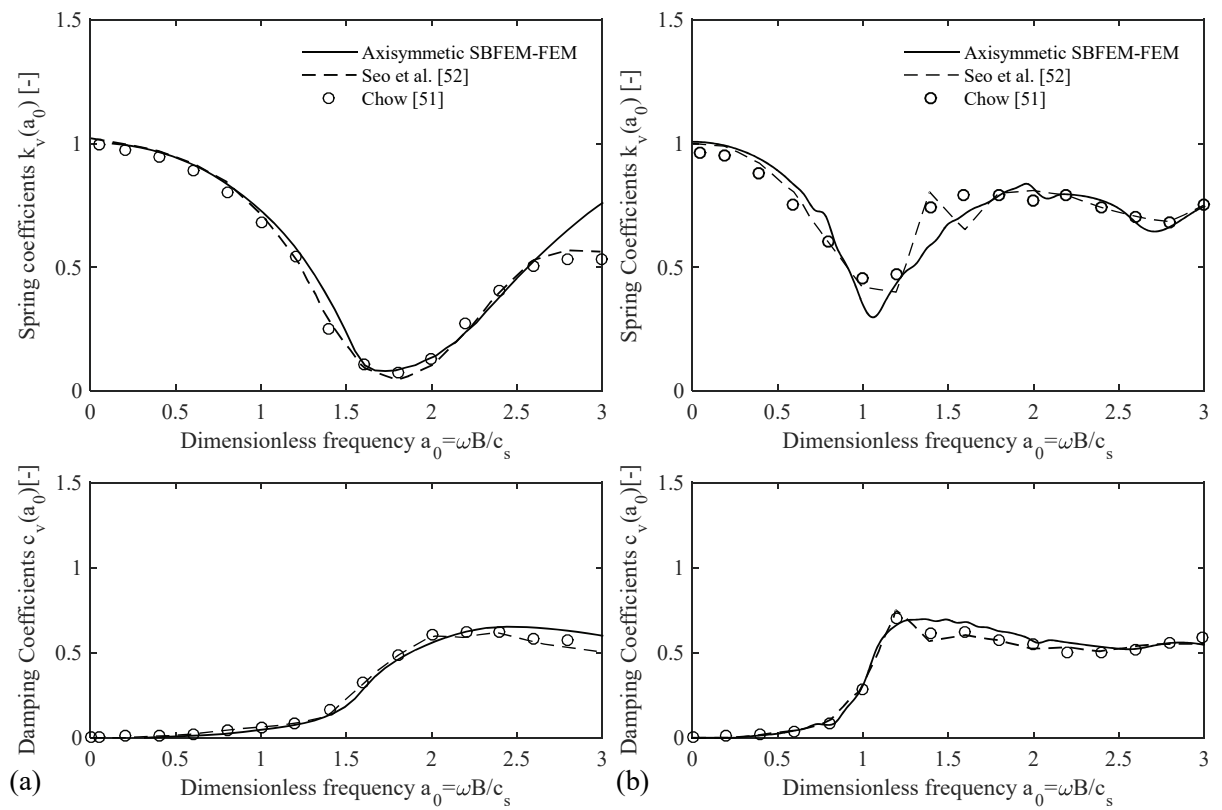

Figure 14 - Vertical impedances of rigid rectangular foundation resting on homogeneous soil layer $(L / B=2, v=0.33, D=0.05)$ (a) $h / B=4$, (b) $h / B=6$

\section{Symbols}
$\xi \quad:$ Scaled radial coordinate
$\omega \quad:$ : Angular frequency
$\sigma \quad:$ Stress
D : Constitutive matrix
u : Displacement
$\varepsilon \quad:$ Strain
L : Differential operator in cylindrical coordinate
$\overline{\mathrm{L}} \quad$ : Equilibrium operator in cylindrical coordinate
$\mathrm{N}(\mathrm{s}) \quad$ : Shape function matrix
$\mathrm{n} \quad$ : Term of Fourier series $(\mathrm{n}>=0)$
$\mathrm{F}_{u}^{s}, \quad \mathrm{~F}_{u}^{a} \quad$ : Symmetric and anti-symmetric trigonometric matrix 
$\mathrm{P}^{s}, \mathrm{P}^{a} \quad$ : Symmetric and anti-symmetric equivalent nodal force

$\mathrm{E}^{i s}, \mathrm{E}^{i a}$ : Symmetric and anti-symmetric coefficient matrix $(0=<\mathrm{i}<=5)$

$\mathrm{M}_{0}^{s}, \mathrm{M}_{0}^{a} \quad$ : Symmetric and anti-symmetric mass matrix

$\mathrm{S}^{s}, \mathrm{~S}^{a} \quad$ : Symmetric and anti-symmetric stiffness matrix

$a_{k}, b_{k} \quad$ : Fourier coefficients

$\mathrm{f}_{k}^{s}, \mathrm{f}_{k}^{a} \quad$ : Symmetric and anti-symmetric loading vector of the discretized line

K : Static stiffness matrix

M : Mass matrix

$\widehat{\mathrm{P}} \quad$ : Nodal force

$\widehat{\mathrm{R}}_{b} \quad$ : Coupling force

$\widehat{\mathrm{P}}_{e x} \quad$ : External loads

$\widehat{\mathrm{P}}_{i} \quad:$ Interior loads

$\widehat{\mathrm{P}}_{\mathrm{b}} \quad$ : Interface loads

$\mathrm{T} \quad$ : Transformation matrix

$a_{0} \quad$ : Dimensionless frequency

G : Shear modulus

$\rho \quad$ : Density

$v \quad$ : Poisson's ratio

$c_{\mathrm{s}} \quad$ : Shear Wave velocity

$\mathrm{K}_{\text {stat }} \quad$ : Static stiffness coefficient

\section{Acknowledgements}

The first author (M.A.) would like to thank professor Carolin Birk from the university of Duisburg-Essen for her brilliant ideas, supportive and respectful supervision during the author's visit in 2017. Dr. Hauke Gravenkamp is to be acknowledged gratefully for his considerable contribution and support in the computer programming.

\section{References}

[1] Kausel E., "Local transmitting boundaries", Journal of engineering mechanics, 114(6), 1011-1027, 1988.

[2] Givoli D., "Non-reflecting boundary conditions: a review", Journal of Computational Physics, 8, 1-29, 1991. 
[3] Givoli D., Numerical methods for problems in infinite domains. vol. 33, Elsevier, 2013.

[4] Tsynkov S.V., "Numerical solution of problems on unbounded domains. a review", Applied Numerical Mathematics, 27(4), 465-532, 1998.

[5] Givoli D., "High-order local non-reflecting boundary conditions: a review", Wave motion, 39(4), 319-326, 2004.

[6] Astley R.J., "Infinite elements for wave problems: a review of current formulations and an assessment of accuracy", International Journal for Numerical Methods in Engineering, 49, 951-976, 2000.

[7] Karabalis D.L., and Mohammadi M., "3-D dynamic foundation-soil-foundation interaction on layered soil", Soil Dynamics and Earthquake Engineering, 17(3), 139 $152,1998$.

[8] Bouchon M., “A simple method to calculate Green's functions for elastic layered media", Bulletin of the Seismological Society of America, 71(4), 959 - 971, 1981.

[9] Schmid G., Willms G., Huh Y., and Gibhardt M., "SSI 2D/3D soil structure interaction: Ein Programmsystem zur Berechnung von Bauwerk-BodenWechselwirkungsproblemen mit der Randelementmethode", Wissenschaftliche Mitteilungen, Nr. 12, SFB 151, Ruhruniversität Bochum, 1988.

[10] Lysmer J., and Waas G., "Shear waves in plane infinite structures", Journal of the Engineering Mechanics Division, 98(EM1), 85-105, 1972.

[11] Kausel E., and Roesset J.M., "Dynamic stiffness of circular foundations", Journal of the Engineering Mechanics Division, 101, 771-785, 1975.

[12] Kausel E., and Peek R., "Dynamic loads in the interior of a layered stratum: An explicit solution”, Bulletin of the Seismological Society of America, 72, 1459-1480, 1982.

[13] Kausel E., "Wave propagation in anisotropic layered media", International Journal for Numerical Methods in Engineering, 23, 1567-1578, 1986.

[14] Seale S.H., and Kausel E., "Point loads in cross-anisotropic layered half spaces", Journal of Engineering Mechanics, 115, 509- 524, 1989.

[15] Kausel E., "Thin-layer method: formulation in the time domain", International Journal for Numerical Methods in Engineering, 37, 927-941, 1994.

[16] Meek J.W., and Wolf J.P., "Cone models for soil layer on rigid rock. II", Journal of Geotechnical Engineering, 118, 686-703, 1992.

[17] Wolf J.P., and Paronesso A., "Lumped-parameter model for a rigid cylindrical foundation embedded in a soil layer on rigid rock", Earthquake Engineering and Structural Dynamics, 21, 1021-1038, 1992.

[18] Wolf J.P., and Preisig M., "Dynamic stiffness of foundation embedded in layered halfspace based on wave propagation in cones", Earthquake Engineering and Structural Dynamics, 32(7), 1075 - 1098, 2003. 
[19] Pradhan P.K., Baidya D.K., and Ghosh D.P., "Dynamic response of foundations resting on layered soil by cone model", Soil Dynamics and Earthquake Engineering, 24(6), 425 $-434,2004$.

[20] Baidya D.K., Muralikrishna G., and Pradhan P.K., "Investigation of foundation vibrations resting on a layered soil system", Journal of Geotechnical and Geoenvironmental Engineering, 132(1), 116 - 123, 2006.

[21] Anam I., and Roësset J.M., "Dynamic stiffness of surface foundations: An explicit solution", International Journal of Geomechanics, 4(3), 216-223, 2004.

[22] Nogami T., Mahbub A.A., and Chen S.H., "A new method for formulation of dynamic responses of shallow foundations in simple general form", Soil Dynamics and Earthquake Engineering, 25(7-10), 679 - 688, 2005.

[23] Gazetas G., "Static and dynamic displacements of foundations on heterogeneous multilayered soils", Géotechnique, 30(2), 159 - 177, 1980.

[24] Andersen L., and Clausen J., "Impedance of surface footings on layered ground", Computers and Structures, 86(1-2), 72 - 87, 2008.

[25] Bouchon M., “A simple method to calculate Green's functions for elastic layered media", Bulletin of the Seismological Society of America, 71(4), 959-971, 1981.

[26] Luco J.E., and Apsel R.J., “On the Green's functions for a layered half-space. Part I”, Bulletin of the Seismological Society of America, 73(4), 909-929, 1983.

[27] Apsel R.J., and Luco J.E., “On the Green's functions for a layered half-space. Part II”, Bulletin of the Seismological Society of America, 73(4), 931-951, 1983.

[28] Birk C., and Behnke R., "A modified scaled boundary finite element method for threedimensional dynamic soil-structure interaction in layered soil", International Journal for Numerical Methods in Engineering, 89(3), 371-402, 2012.

[29] Wolf J.P., Foundation Vibration Analysis Using Simple Physical Models, PrenticeHall, Englewood Cliffs NJ, 1994.

[30] Gazetas G., "Analysis of machine foundation vibrations: State of the art", Soil Dynamics and Earthquake Engineering, 2(1), 2 - 42, 1983.

[31] Wolf J.P., The Scaled Boundary Finite Element Method, Wiley \& Sons: Chichester, 2003.

[32] Genes M.C., and Kocak S., "Dynamic soil-structure interaction analysis of layered unbounded media via a coupled finite element/ boundary element/scaled boundary finite element model", International Journal for Numerical Methods in Engineering, 62(6), 798-823, 2005.

[33] Genes M., and Kocak S., "A combined finite element based soil-structure interaction model for large-scale systems and applications on parallel platforms", Engineering Structures, 24(9), 1119-1131, 2002. 
[34] Genes M.C., "Dynamic analysis of large-scale SSI systems for layered unbounded media via a parallelized coupled finite element/ boundary-element/scaled boundary finite-element model", Engineering Analysis with Boundary Elements, 36(5), 845-857, 2012.

[35] Yaseri A., Bazyar M., and Hataf N., "3D coupled scaled boundary finite-element/finiteelement analysis of ground vibrations induced by underground train movement", Computers and Geotechnics, 60, 1-8, 2014.

[36] Syed N.M., and Maheshwari B.K., Improvement in the computational efficiency of the coupled FEM-SBFEM approach for 3D seismic SSI analysis in the time domain. Computers and Geotechnics, 67, 204-212, 2015.

[37] Rahnema H., Mohasseb S., and JavidSharifi B., "2-D soil-structure interaction in time domain by the SBFEM and two non-linear soil models", Soil Dynamics and Earthquake Engineering, 88,152-175, 2016.

[38] Li B., Cheng L., Deeks A.J., and Teng B., "A modified scaled boundary finite-element method for problems with parallel side-faces. Part I. Theoretical developments", Applied Ocean Research, 27(4 - 5), 216 - 223, 2005.

[39] Lin G., Du J., and Hu Z., "Dynamic dam-reservoir interaction analysis including effect of reservoir boundary absorption", Science in China Series E: Technological Sciences, $50,1-10,2007$.

[40] Doherty J.P, and Deeks A.J., "Scaled boundary finite-element analysis of a nonhomogeneous elastic half-space", International journal for numerical methods in engineering, 57(7), 955-973, 2003.

[41] Aslmand M., Kani I.M., Birk C., Gravenkamp H., Krome F., Ghadi M.E., "Dynamic soil-structure interaction in a 3D layered medium treated by coupling a semi-analytical axisymmetric far field formulation and a 3D finite element model" Soil Dynamics and Earthquake Engineering, 115, 531-544, 2018.

[42] Sommerfeld A. Partial Differential Equations in Physics, Academic Press: New York, 1949.

[43] Guzina B.B., Fata S.N., and Bonnet M., "On the stress-wave imaging of cavities in a semi-infinite solid", International Journal of Solids and Structures, 40, 1505-1523, 2003.

[44] Madyarov A.I., and Guzina B.B., "A radiation condition for layered elastic media", Journal of Elasticity, 82, 73-98, 2006.

[45] Song C., and Wolf J.P., "The scaled boundary finite-element method - alias consistent infinitesimal finite-element cell method - for elastodynamics", Computer Methods in Applied Mechanics and Engineering, 147, 329-355, 1997.

[46] Laub AJ., "A Schur method for solving algebraic Riccati equations", IEEE Transactions on Automatic Control 1979, 24(6):913-921.

[47] Bartels R.H., and Stewart G.W., "Solution of the equation $\mathrm{AX}+\mathrm{XB}=\mathrm{C}$ ", Communications of the ACM 15, 9, 820-826, 1972. 
[48] Ansys computer program, Ansys Inc. Version 18.0, 2016.

[49] Sun E.Q. Shear locking and hourglassing in msc nastran, abaqus, and ansys, Msc software users meeting, 2006.

[50] Emperador J.M., and Dominguez J., "Dynamic response of axisymmetric embedded foundations", Earthquake Engineering and Structural Dynamics, 18(8), 1105 - 1117, 1989.

[51] Chow Y., "Vertical vibration of three-dimensional rigid foundations on layered media", Earthquake engineering \& structural dynamics, 15(5), 585-594, 1987.

[52] Seo C.G., Yun C.B., and Kim J.M., "Three-dimensional frequency-dependent infinite elements for soil-structure interaction", Engineering Structures, 29(11), 3106-3120, 2007. 